\title{
Influence of sperm movement parameters on human sperm-oolemma fusion
}

\author{
J. Ph. Wolf ${ }^{1}$, D. Feneux ${ }^{2}$, B. Ducot ${ }^{3}$, D. Rodrigues ${ }^{1}$ and P. Jouannet ${ }^{1}$ \\ ${ }^{1}$ Laboratoire de Biologie de la Reproduction, Histologie, Embryologie, Université Paris V, Hôpital Cochin, \\ 123 Bd. Port Royal 75014 Paris, France; ${ }^{2}$ Laboratoire de Biologie de la Reproduction et du Développement \\ - Histologie, Embryologie, Cytogénétique, Hôpital Bicêtre, 78, rue du Général Leclerc 94275, Le Kremlin. \\ Bicêtre, France; and ${ }^{3}$ INSERM U 292. Hôpital Bicêtre, 78, rue du Général Leclerc 94275, Le Kremlin, \\ Bicêtre, France
}

Flagellar dyskinesia is characterized by abnormal sperm movement parameters and a negative sperm mucus penetration test. It is associated with structural pathologies of the axonemal complex (lack of outer dynein arms), of the periaxonemal complex (sliding spermatozoa and periaxonemal dyskinesia), or of both structures (short flagella). Even during in vitro fertilization, dyskinesia prevents the spermatozoon from getting through the egg vestment. However, in some cases, fertilization has been achieved using subzonal insemination. Flagellar dyskinesia is therefore an interesting model for investigating the role of sperm movement in the fusion process between the spermatozoon and the oolemma. Thirty-one patients requiring assisted fertilization were included in the study. Fifteen had spermatozoa in which the flagellum lacked outer dynein arms, 11 had anomalies of the periaxonemal complex (five with sliding spermatozoa and six with periaxonemal dyskinesia) and five had spermatozoa with short flagella. Seven men who produced spermatozoa with normal movement were selected as controls. Movement was evaluated using a computerassisted analyser, and penetration was assessed using zona-free hamster eggs. At $37^{\circ} \mathrm{C}$, in semen, the dyskinetic spermatozoa had reduced straight line and curvilinear velocity and lateral head displacement compared with controls $(P<0.01)$. In the Percoll-selected sperm suspension, the only difference was that spermatozoa with periaxonemal anomalies maintained a narrow lateral head displacement compared with the controls $(P<0.001)$. After $3 \mathrm{~h}$ of incubation at $37^{\circ} \mathrm{C}$, the lateral head displacement of dyskinetic spermatozoa had not changed, while that of the controls showed a significant increase $(4.5$ to $5.6 \mu \mathrm{m} ; P<0.05)$. The results from the sperm penetration assay for the spermatozoa lacking outer dynein arms were lower than those of the controls $(47 \%$ versus $77 \% ; P<0.05)$ and the results for sliding spermatozoa and spermatozoa with periaxonemal dyskinesia were even lower $(25 \%$ and $34 \%$, respectively; $P<0.01$ ). The fertilization rates after subzonal insemination were $46.5 \%$ for spermatozoa lacking outer dynein arms, $36.1 \%$ for spermatozoa with short flagella, $24.8 \%$ for sliding spermatozoa and $17.3 \%$ for spermatozoa with periaxonemal dyskinesia. There was a significant correlation between the curvilinear velocity of the Percoll-selected sperm suspensions and their fertilization rates after subzonal insemination $(r=0.5 ; P<0.05)$ and their sperm penetration assays $(r=0.7 ; P<0.001)$. The data provide evidence that sperm velocity is correlated with the ability to fuse with the oolemma.

\section{Introduction}

In addition to the percentage of motile forms, sperm movement parameters are critical to the fertilizing ability of spermatozoa. Indeed, adequate lateral head displacement of the spermatozoon is required for it to penetrate the cervix (Aitken et al., 1986) and abnormal movement prevents spermatozoa from getting through the cervical mucus (Aitken et al., 1985, 1986;

Manuscript received 7 March 1995
Mortimer et al., 1986; Katz et al., 1989). In cases of flagellar dyskinesia, standard in vitro fertilization (IVF) techniques resulted in low or even nil fertilization rates (Wolf et al., 1993). Different aetiologies have been described for flagellar dyskinesia (Jouannet et al., 1983; Escalier et al., 1984, 1990; Feneux et al., 1985). Diagnosis of flagellar dyskinesia is based on three criteria: (1) on the demonstration of abnormal sperm movement by computer-assisted semen analysis; (2) on tests of functional sperm movement inefficiency, such as negative cervical mucus penetration tests (David et al., 1993); and (3) by electron 
microscope examination of the flagella, to identify structural abnormalities. Dyskinesia results from either a modification of the axonemal complex, such as the lack of outer dynein arms (Jouannet et al., 1983; Escalier et al., 1984) or of the periaxonemal structures such as sliding spermatozoa (Feneux et al., 1985; Serres et al., 1986), or other specific periaxonemal dyskinesia. When both the axonemal and the periaxonemal structures are truncated, the result is the syndrome of the short flagella. However, sperm movement parameters are affected differently by the various types of flagellar dyskinesia and dyskinesia does not prevent the spermatozoon from fusing with the oolemma. It has been shown that sperm penetration performed with spermatozoa lacking outer dynein arms is half of that of controls (Courtot et al., 1985). Several cases of pregnancy have been reported that resulted from subzonal insemination with spermatozoa lacking outer dynein arms where previous IVF attempts had failed (Wolf et al., 1993). In addition, 52 of 90 human oocytes were fertilized when subzonal insemination was performed compared with none of 23 oocytes when standard IVF was used (Wolf et al., 1993). Subzonal insemination is, therefore, a useful model for studying the influence of sperm movement parameters on the spermoolemma fusion process. In the present work the movement parameters of spermatozoa were studied in semen, in Percollselected sperm suspension and after incubation for $3 \mathrm{~h}$ in a capacitation medium. The correlation between these data and the fertilizing ability of the spermatozoa was evaluated using sperm penetration assays and their fertilization rates after subzonal insemination.

\section{Materials and Methods}

\section{patients}

Thirty-one men with infertility characterized by extremely low fertilization rates during IVF or, more often, complete fertilization failure and a negative cervical mucus penetration test were included in the study. Spermatozoa from these patients underwent a movement analysis using a computer assisted sperm analysis system. When abnormal sperm movement parameters were found (Jouannet et al., 1983; Feneux et al., 1985). spermatozoa were further analysed by electron microscopy. Spermatozoa from fifteen patients lacked outer dynein arms; on each section of the flagella at least six outer dynein arms were lacking. Eleven of these patients presented this anomaly in $100 \%$ of their spermatozoa, and the remaining four had at least $60 \%$ of their spermatozoa with this abnormality (Fig. 1). Eleven patients presented periaxonemal anomalies. The periaxonemal structures of these patients were modified without specific associated axonemal anomalies. Among these anomalies, modifications in the order of termination of the dense fibres, or of their number, and malformation of the longitudinal columns were found: in this group with periaxonemal anomalies, five patients had sliding spermatozoa with an abnormal order of termination of the dense fibres along the principal piece and often an abnormal number or position of their longitudinal columns. Movement of sliding spermatozoa is characterized by a very small amplitude of the flagellar

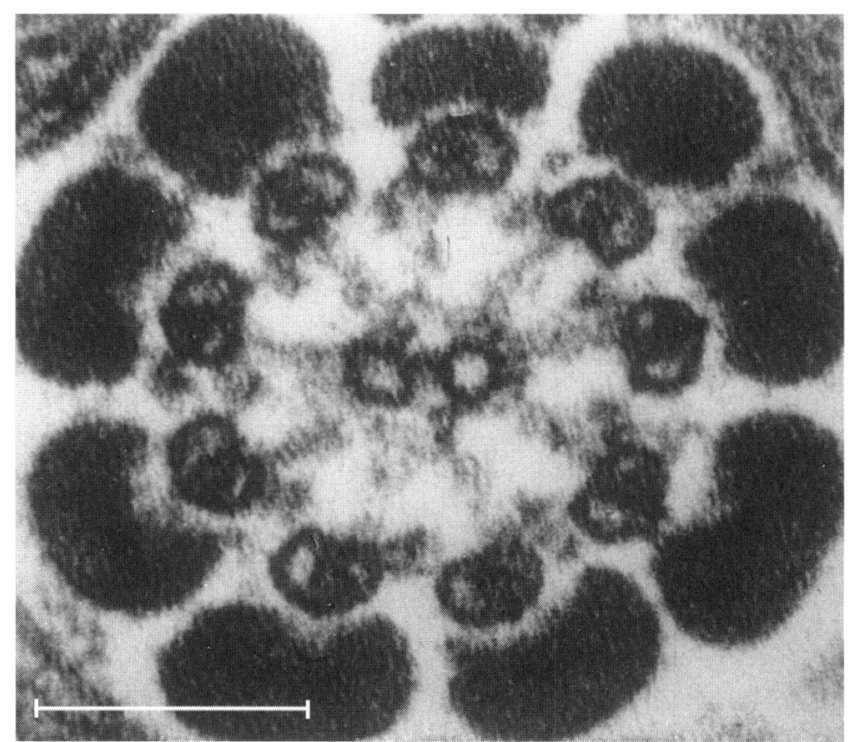

Fig. 1. Electron micrograph of the principal piece of human sperm flagella. The outer dynein arms are absent. Scale bar represents $1 \mu \mathrm{m}$.

bend and a discordance between the high beat frequency and the low cell rotation frequency. The remaining six patients in this group presented other types of periaxonemal dyskinesia (Fig. 2) with unilateral thickening of the fibrous sheet instead of individual column and anomalies of the dense fibres. These anomalies are associated with a slow development and propagation of the flagellar wave. The five other patients in the study had short flagella syndrome characterized by a very short principal piece and disorganization of the axonemal and peri-axonemal structures. For all these patients, the relevant anomaly was found in at least $60 \%$ of their spermatozoa. Sixty-five attempts using subzonal insemination were carried out involving $55 \mathrm{I}$ oocytes.

Seven men were selected for the control group. They belonged to a group of patients with unexplained IVF failure who were included in the subzonal insemination programme. Their spermatozoa had normal movement parameters, presented a positive cervical mucus penetration test and a zona-free hamster egg penetration test result of over $60 \%$.

\section{Processing of spermatozoa}

Ejaculates were collected by masturbation at the laboratory after 3 days of sexual abstinence. An aliquot was kept for determination of semen characteristics and sperm movement analysis using computer-aided sperm analysis. The spermatozoa were layered over a two-step (47.5\% and 95\%) Percoll gradient $(0.5 \mathrm{ml}$ per layer $)$ and centrifuged for $20 \mathrm{~min}$ at $300 \mathrm{~g}$. The pellet containing the highly motile sperm fraction was removed and washed twice by centrifugation for $10 \mathrm{~min}$ at $600 \mathrm{~g}$ with $5 \mathrm{ml} \mathrm{B2}$ medium (Api System, Marcy l'Etoile). The final sperm pellets were resuspended in 0.2-0.5 ml B2 medium. The sperm movement parameters were then determined again 

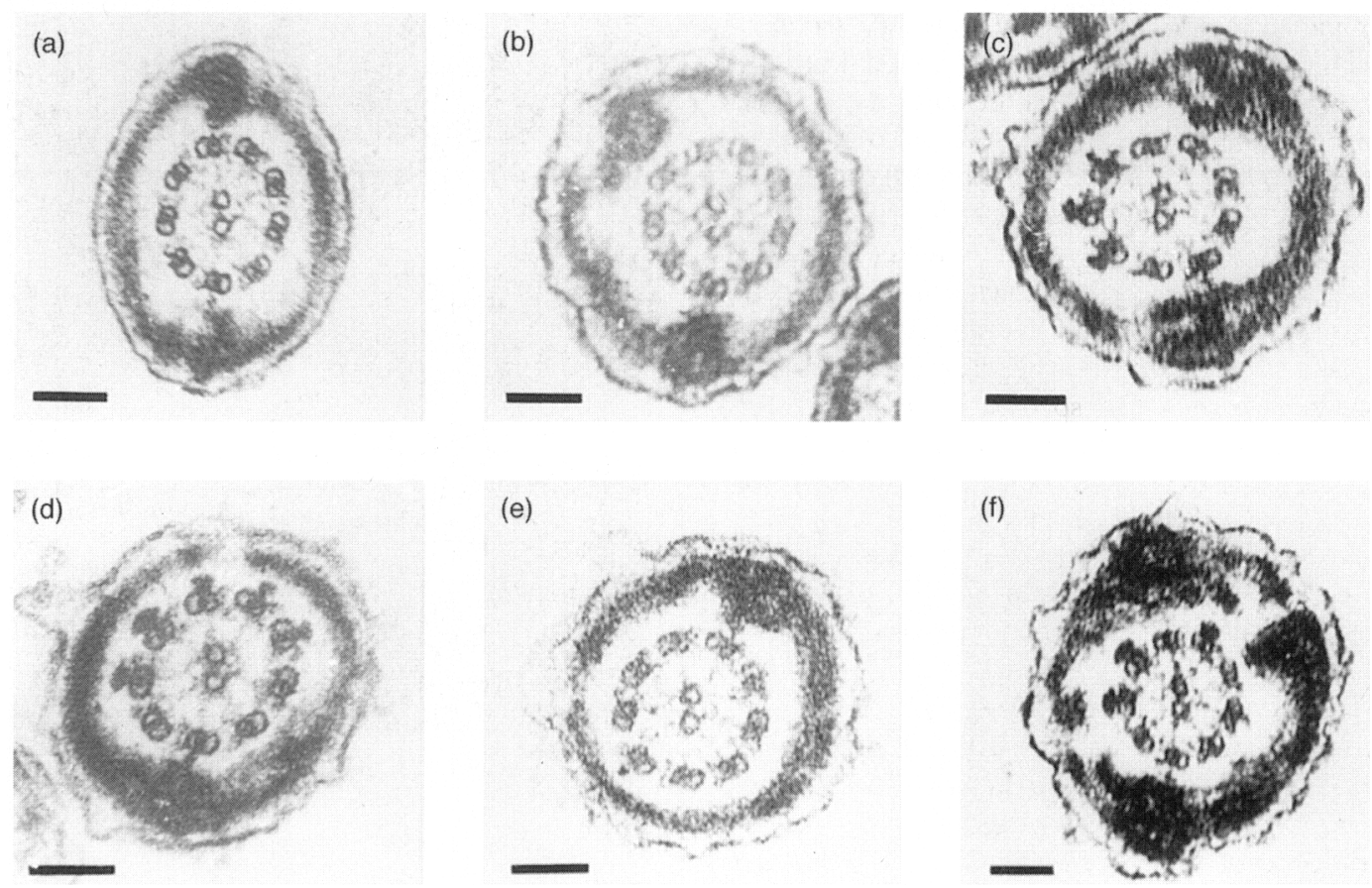

Fig. 2. Electron micrographs of human sperm flagella showing periaxonemal anomalies. (a) Normal position of the two longitudinal columns; (b) and (c) abnormal position of the longitudinal column; (d) and (e) only one column is present, in normal (d) and abnormal (e) position. (f) Thickening of the fibrous sheath considered as a third column. Scale bars represent $0.1 \mu \mathrm{m}$.

in the Percoll selected sperm suspensions and after incubation for $3 \mathrm{~h}$ at $37^{\circ} \mathrm{C}$ in $5 \% \mathrm{CO}_{2}$ in air.

\section{Analysis of spermatozoa}

The percentage of motile spermatozoa was estimated under a microscope $(\times 200)$ using a Bausch and Lomb CAT $312995 \times 2$ objective lens and a CCD camera. For morphological analysis, 100 spermatozoa were examined $(\times 1000)$ on a Shorr-stained smear (Shorr Staining: Merck, Darmstadt) and classified according to the criteria of David et al. (1975). The motility of the aliquots of Percoll-selected sperm suspensions was analysed at $37^{\circ} \mathrm{C}$ using a computer-assisted semen analyser (HTM 2030 version 7.2; Hamilton-Thorn Research, Danvers, MA). The following settings were used: frame at frame rate, 20 at $25 \mathrm{~s}^{-1}$; minimum contrast, 8 ; minimum size, 10; low and high size gates, 0.6 and 1.8, respectively; low and high intensity gates, 0.6 and 1.7, respectively; non-motile head size, 7; non-motile intensity, 110; medium average path velocity value, $25 \mu \mathrm{m} \mathrm{s}^{-1}$; low average path velocity value, $10 \mu \mathrm{m} \mathrm{s}^{-1}$; slow cell motile, yes; percentage progressive cells equated with a straightness of $>80 \%$, regardless of average path velocity. An aliquot of the sperm suspension was diluted to $10^{6}$ cells $\mathrm{ml}^{-1}$ with B2 medium and loaded into a $200 \mu \mathrm{m}$ deep flat glass microcapillary tube (Vitro Dynamics, Rockaway, NJ) that was transferred to the analyser at $37^{\circ} \mathrm{C}$. At least 150 motile spermatozoa were scored to obtain mean values for the following movement parameters: curvilinear velocity, straight line velocity, amplitude of lateral head displacement, linearity (straight line velocity/curvilinear velocity), and beat cross frequency.

\section{Electron microscope study of sperm flagella}

Semen samples were fixed for $1 \mathrm{~h}$ in $2.5 \%(\mathrm{v} / \mathrm{v})$ glutaraldehyde in $0.1 \mathrm{~mol}$ Sörensen's buffer $1^{-1}$, washed in buffer supplemented with $4 \%(\mathrm{w} / \mathrm{v})$ sucrose before being embedded in $2 \%$ agar. After dehydration in a graded series of ethanol, small pieces of agar were embedded in Araldite. Sections $(0.05 \mu \mathrm{m})$ were stained with uranyl acetate $(4 \%(\mathrm{w} / \mathrm{v})$ in $70 \%(\mathrm{v} / \mathrm{v})$ ethanol, for $20 \mathrm{~min}$ ) and $10 \%(\mathrm{w} / \mathrm{v})$ lead citrate (10 min) and examined in a Siemens Elmiskop CT 150 transmission electron microscope.

The ultrastructural assessment was made on transverse sections through the sperm tails which provide quantitative observations on the various axonemal and periaxonemal structures, and on longitudinal sections, which provide nonquantitative observations, such as the extent of the anomaly along the tail and possible associations. Dynein arms were considered to be absent from a section if at least six of the nine elements were missing.

\section{Zona-free hamster egg penetration assay}

Sexually mature, female golden hamsters were superovulated by an injection of 40 iu pregnant mares' serum gonadotrophin (Folligon: Intervet, Angers) and ovulation was induced with 40 iu hCG (Chorulon: Intervet, Angers) $48 \mathrm{~h}$ later, beginning on day $\mathrm{I}$ of the oestrous cycle. The oocytes were collected in BWW medium supplemented with $3.0 \mathrm{mg}$ HSA ml ${ }^{-1}$ fraction 5 (Sigma, St Louis, MO). Cumulus cells were removed by brief exposure to $0.1 \%(\mathrm{w} / \mathrm{v})$ hyaluronidase type III (Sigma). Zonae pellucidae were removed using 0.1\% bovine trypsin $(0.1 \% \mathrm{w} / \mathrm{v})$ (Sigma). 


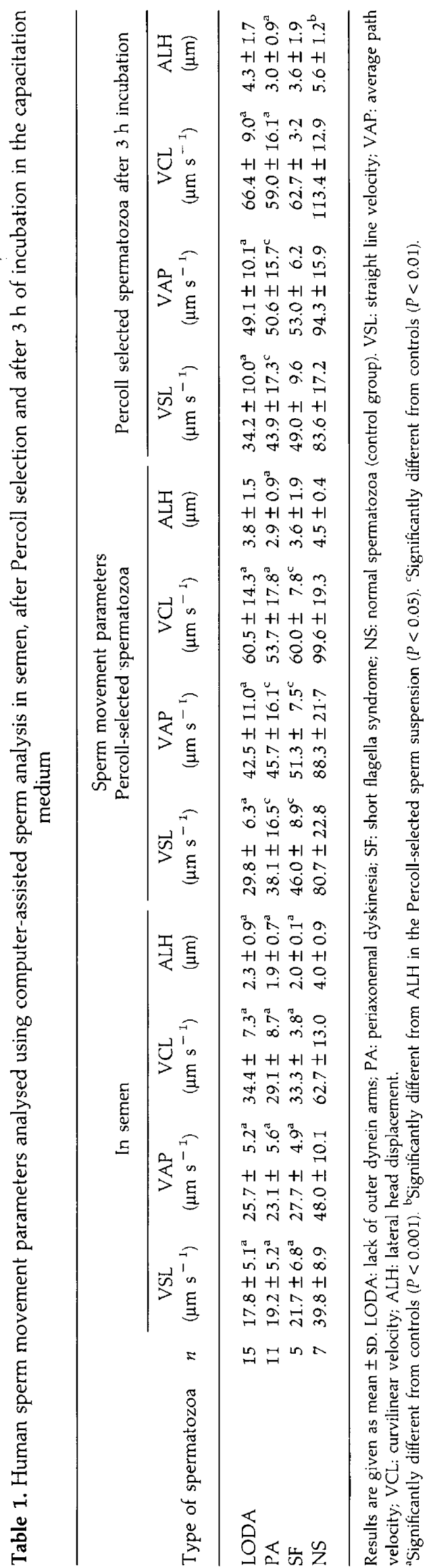


Semen was collected on the day before the test and was allowed to liquefy for $30 \mathrm{~min}$ at $37^{\circ} \mathrm{C}$. After determination of the sperm characteristics, it was layered over a two-step Percoll gradient $(95 \%$ and $47.5 \%$ ) and centrifuged for $20 \mathrm{~min}$ at $300 \mathrm{~g}$. BWW medium, with an osmolarity of $410 \mathrm{mosmol} \mathrm{kg}{ }^{-1}$, was used for washing and recovery of the pellet. Volume, number, motility and morphology of spermatozoa were assessed in the Percoll-selected sperm suspension. The suspension then underwent capacitation for $20 \mathrm{~h}$ at $20^{\circ} \mathrm{C}$ in air before use. Five oocytes were incubated for $3 \mathrm{~h}$ per $30 \mu \mathrm{l}$ drop containing $2.5 \times 10^{6}$ motile spermatozoa $\mathrm{ml}^{-1}$ under mineral oil. Incubation was performed in air with $5 \% \mathrm{CO}_{2}$ and thirty oocytes were used per test. At the end of the $3 \mathrm{~h}$ period, the oocytes were washed, transferred to a clean microscope slide and compressed to a depth of approximately $30 \mu \mathrm{m}$ with a $22 \mathrm{~mm} \times 22 \mathrm{~mm}$ coverslip mounted on four paraffin wax supports. Control incubations were carried out using a pool of frozen donor spermatozoa and usually resulted in more than $60 \%$ of the oocytes containing decondensed sperm heads.

\section{Oocyte preparation}

Superovulations were performed in the Gynaecological Departments of the Hôpital A. Beclere (R. Frydman, Clamart), of the Hôpital International de la Cité Universitaire (Paris) (Ph. Granet and G. Sarrot, Paris), of the Hôpital Saint Vincent de Paul (Paris) (S. Epelboin), and of the Hôpital Jean Verdier (Bondy) (J. N. Hugues). Follicular growth was stimulated by human menopausal gonadotrophins (hMG) associated with a GnRH agonist. Ovulation was induced by administration of $10000 \mathrm{iu} \mathrm{hCG}$ and the oocytes were collected $36 \mathrm{~h}$ later using a transvaginal ultrasound procedure. The cumuli were washed and placed individually in a $30 \mu \mathrm{l}$ drop of $\mathrm{B} 2$ medium under equilibrated oil. Cumulus cells were removed by exposure of the oocytes to $0.1 \%(\mathrm{w} / \mathrm{v})$ hyaluronidase (Type III, Sigma) in B2 medium, and the nuclear status of the oocytes was assessed.

\section{Subzonal insemination}

Subzonal insemination was performed as described by Wolf et al. (1993) on oocytes at metaphase II, 6-8 h after collection. Two Narishige micromanipulators and one inverted IMT-2 microscope (Olympus) were suitable for carrying out the procedure. The number of spermatozoa that had to be microinjected to obtain diploid fertilization was not known; therefore, between one and 16 spermatozoa were chosen randomly from among those with forward progressive movement and normal morphology and microinjected into the perivitelline space.

\section{Analysis criteria}

Oocytes were checked 16-18 h after subzonal insemination for evidence of fertilization. Oocytes exhibiting two pronuclei were considered as normally fertilized and the zygotes were kept in culture for a further $24 \mathrm{~h}$. Only regularly cleaved embryos were transferred into the uterus using a Frydman catheter (CCD, Paris).

\section{Statistical analysis}

The relationship between the IVF success rates, the results of the sperm penetration assay and the sperm characteristics were examined by Spearman's rank correlation coefficients using the Statworks package. The results of the sperm penetration assay and comparisons between the movement parameter values for the different types of dyskinesia were performed using the Mann-Whitney non-parametric test.

\section{Results}

The sperm concentration from patients with flagellar dyskinesia did not differ from that of the controls. All the dyskinetic sperm samples had a reduced percentage of motile forms of normal forms (except those exhibiting periaxonemal dyskinesia) compared with the controls.

\section{Effect of Percoll selection on sperm parameters}

The velocity parameters of normal spermatozoa increased after Percoll selection in the capacitation medium when compared with their values in semen (Table 1). The straight line velocity and average path velocity doubled while the curvilinear velocity showed a $60 \%$ increase, from 62.7 to $99.6 \mu \mathrm{m} \mathrm{s}^{-1}$. However, the lateral head displacement value did not change significantly ( 4.0 and $4.5 \mu \mathrm{m}$, respectively). During capacitation in the inseminating medium, the velocity parameters of normal spermatozoa showed a moderate increase, while the lateral head displacement showed a significant change from 4.5 to $5.6 \mu \mathrm{m}(P<0.05)$. All types of dyskinetic spermatozoa had lower velocities than did the controls $(P<0.001)$ in semen. They also had significantly smaller lateral head displacements $(P<0.05)$. The velocity parameters of all the dyskinetic sperm increased, but remained significantly different from the control values $(P<0.01)$, after Percoll selection. After Percoll selection, lateral head displacement values for spermatozoa lacking outer dynein arms and spermatozoa with short flagella increased such that they were no longer significantly different from the value for normal spermatozoa. Spermatozoa with periaxonemal anomalies showed little variation in their lateral head displacement which remained significantly smaller than that of the controls $(2.9 \mu \mathrm{m}$ versus $4.5 \mu \mathrm{m} ; P<0.001)$.

\section{Effect of incubation period on Percoll-selected spermatozoa}

After $3 \mathrm{~h}$ incubation, the velocity of the spermatozoa with short flagella increased slightly while the velocity parameters for spermatozoa lacking outer dynein arms and with periaxonemal dyskinesia increased between 10 and $15 \%$, but remained significantly different from control values $(P<0.01)$. Lateral head displacement values of spermatozoa with periaxonemal anomaly and with short flagella did not change but that for spermatozoa lacking outer dynein arms showed a $13 \%$ increase.

\section{Sperm penetration assays}

The results of the sperm penetration tests using zona-free hamster oocytes are presented (Table 2). Spermatozoa from 
Table 2. Sperm penetration assay of dyskinetic and control human spermatozoa

\begin{tabular}{lrcc}
\hline Type of spermatozoa & $n$ & Mean \pm SD & Range \\
\hline Short flagella & 1 & 22 & \\
Sliding spermatozoa & 4 & $25.3 \pm 9.3$ & $12-33^{*}$ \\
Periaxonemal dyskinesia & 6 & $34.3 \pm 13.2$ & $16-56^{*}$ \\
Lacking outer dynein arms & 13 & $47.0 \pm 26.5$ & $8-93^{* *}$ \\
Controls & 7 & $77.7 \pm 20.2$ & $44-100$ \\
& & & \\
\hline
\end{tabular}

Significantly different from controls $\left({ }^{*} P<0.01 ;{ }^{* *} P<0.05\right)$.

patients with short flagella syndrome do not migrate well through the Percoll gradient; therefore, only one patient of this group had the sperm penetration test. Sliding spermatozoa and spermatozoa with periaxonemal dyskinesia had the most impaired scores compared with controls $(P<0.01)$. The difference between the results of the sperm penetration tests for spermatozoa lacking outer dynein arms and the controls was less but still significant $(P<0.05)$. There was a correlation between the fertilization rate after subzonal insemination and the results of sperm penetration test for dyskinetic sperm ( 62 cycles, $r=0.3 ; P<0.05$ ). The correlation for spermatozoa with periaxonemal anomalies was higher $(9$ cycles, $r=0.7 ; P<0.05)$. Analysis of the correlation between the sperm movement parameters from dyskinetic and control spermatozoa and their sperm penetration results showed that the curvilinear velocity of Percoll-selected suspensions was the most significant variable $(r=0.7 ; P<0.001)$.

\section{Fertilization rates}

The overall fertilization rate of the 62 attempts using subzonal insemination was $41.8 \%$. The rate varied from $31.3 \%$ for spermatozoa with periaxonemal anomalies to $43.7 \%$ for spermatozoa lacking outer dynein arms. The diploid fertilization rate was almost half the fertilization rate and followed the same pattern. Ten pregnancies resulted from 49 embryo transfers, of which four ended in early termination and six resulted in healthy babies. The correlation between the fertilization rate after subzonal insemination for all types of flagellar dyskinesia and all sperm movement parameters when evaluated in the Percoll-selected spermatozoa were significant for curvilinear velocity $(r=0.5 ; P<0.05)$ (Fig. 3) and lateral head displacement $(r=0.4 ; P<0.05)$. When evaluated after $3 \mathrm{~h}$ of incubation the correlations were curvilinear velocity $(r=0.5$; $P<0.002)$, average path velocity $(r=0.3 ; P<0.05)$ and lateral head displacement $(r=0.3 ; P<0.05)$.

\section{Discussion}

This study provides an analysis of the movement parameters of dyskinetic spermatozoa in semen, after Percoll selection and after $3 \mathrm{~h}$ of incubation in the capacitation medium used for insemination. The results indicate a correlation between fertilization rates after subzonal insemination and movement parameters of dyskinetic spermatozoa.
Several characteristics are common to all types of dyskinesia. In semen, movement parameters are reduced. A significant reduction was observed in sperm velocity (straight line, curvilinear and average path velocities) as well as lateral head displacement. All the values of their movement parameters increased when the spermatozoa were in the capacitation medium after Percoll selection, although they still remained lower than control values. It is significant that the movement parameters of dyskinetic spermatozoa did not improve during the first $3 \mathrm{~h}$ of incubation contrary to the situation for normal spermatozoa for which the value of lateral head displacement increased from a mean of $4.5 \mu \mathrm{m}$ to $5.6 \mu \mathrm{m}$. All the patients in the study were infertile and had failed to achieve pregnancy with standard IVF, justifying the use of subzonal insemination. It has been shown that for patients with autoimmune sperm antibody, increases in values of lateral head displacement during incubation are linked to the ability of spermatozoa to pass through the egg vestment (Zouari et al., 1993). With dyskinetic spermatozoa the absence of an increase in lateral head displacement is also associated with poor IVF results.

Despite low movement parameters, gamete fusion can occur when spermatozoa are delivered close to the oolemma. Gamete fusion has already been reported with spermatozoa lacking dynein arms using subzonal insemination (Wolf et al., 1993). In the present report evidence is provided that the ability of spermatozoa to fuse with the oocyte is significantly correlated with the curvilinear velocity of the Percoll-selected spermatozoa. Interestingly, lateral head displacement, which has been shown to be the most important criterion for sperm fertilizing ability during standard insemination (Zouari et al., 1993) has less significance for the sperm-oolemma interaction. These results confirm, in a human-human system, the relation between sperm movement parameters and fusiogenic ability reported by Aitken et al. (1994) in the human-hamster system. The movement characteristic identified in their study as being of most prognostic value for the sperm-oocyte fusion was the average path velocity and not the curvilinear velocity as in the study reported here. The difference between both these movement parameters was not significant in the study by Aitken et al. (1994); this discrepancy is probably due to the use of microcapillary tubes in our analysis of the sperm movement parameters instead of Microcell and Makler chambers. The depth of these devices is $200 \mu \mathrm{m}, 20 \mu \mathrm{m}$ and $10 \mu \mathrm{m}$, respectively.

The mechanism underlying this association between curvilinear velocity and fertilization rate after subzonal insemination is unknown. The association may indicate that sperm movement is necessary for the sperm-oolemma fusion process. In mice, sperm adhesion treatment in which spermatozoa were held against the ooplasmic membrane after subzonal insemination resulted in a significantly increased fertilization rate (Kobayashi et al., 1992). However, applying the sperm adhesion treatment to immotile spermatozoa did not result in fertilization. This suggests, at least in mice, that sperm motility may be involved in fertilization even after adhesion of the sperm head with the oocyte membrane. This is consistent with two other studies also performed in mice. In the first study, Barg et al. (1986) showed that immobilized spermatozoa do not fertilize the oocyte. In the second study, Klemm and Engel (1991) showed that the microinjection of immotile spermatozoa resulted in phagocytosis rather than in sperm-oocyte fusion. 

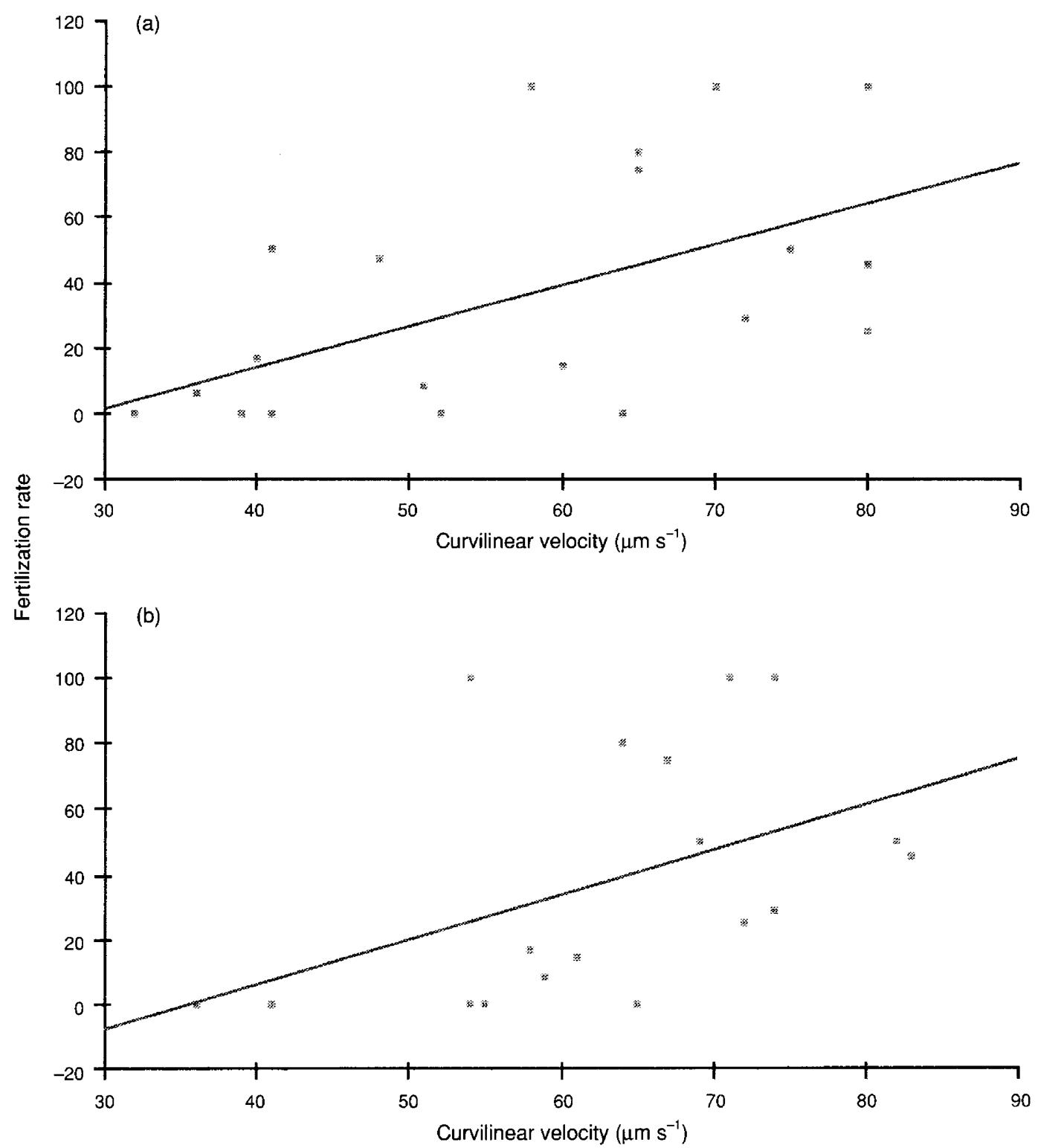

Fig. 3. Correlation between fertilization rates after subzonal insemination and curvilinear velocity. The curvilinear velocity is (a) measured in the Percoll-selected sperm suspension $\left(\mathrm{VCL}_{\mathrm{o}}\right)$ and $(\mathrm{b})$ measured again after incubation for $3 \mathrm{~h}$ in the capacitating medium $\left(\mathrm{VCL}_{3}\right)$. The lines represent the regression lines. Subzonal inseminations performed with less than five oocytes were not included. Fertilization rate: percentage of microinjected oocytes.

However, fertilization of human eggs with immotile spermatozoa has been reported (Bongso et al., 1989), and immotile spermatozoa from men with Kartegener's syndrome penetrate zona-free hamster oocytes (Aitken et al., 1983). Preliminary investigations performed in our laboratory using subzonal insemination with spermatozoa with flagellar dyskinesia linked to metabolic disorders (results not shown) indicated that they almost never fertilized despite high curvilinear velocities. All these data suggest that the relationship between curvilinear velocity and ability of spermatozoa to fuse with the oocyte is not linked to frequency of collisions with the oocyte. We speculate that curvilinear velocity is indicative of the ability of the spermatozoon to fuse. Curvilinear velocity is the result of the forward progression and of the lateral head displacement. Since lateral head displacement represents the propulsive strength of the spermatozoa (David et al., 1981), the ability of a spermatozoon to increase its lateral head displacement in the capacitation medium may reflect the adaptation of its metabolic pathway to the fertilizing conditions, and this adaptation is not possible for dyskinetic spermatozoa.

This work was supported by grant EA 1606 from DRED and grant 91006 from Direction de la Recherche Clinique-AP.

\section{References}

Aitken JR, Ross A and Lees MM (1983) Analysis of sperm function in Kartagener's syndrome Fertility and Sterility 40 696-698

Aitken RJ, Sutton M, Warner P and Richardson DW (1985) Relationship between the movement characteristics of human spermatozoa and their ability to 
penetrate cervical mucus and zona-free oocytes journal of Reproduction and Ferfility 73 441-449

Aitken RJ, Warner PE and Reid C (1986) Factors influencing the success of sperm-cervical mucus interaction in patients exhibiting unexplained infertility Journal of Andrology 7 3-10

Aitken J, Buckingham D and Harkiss D (1994) Analysis of the extent to which sperm movement can predict the results of ionophore-enhanced functional assays of the acrosome reaction and sperm-oocyte fusion Human Reproduction 9 1867-1874

Barg PE, Wahrman MZ, Talansky BE and Gordon JW (1986) Capacitated, acrosome reacted but immotile sperm, when microinjected under the mouse zona pellucida, will not fertilize the oocyte Journal of Experimental Zoology 237 365-374

Bongso TA, Sathananthan AH, Wong PC, Ratnam SS, Ng SC, Anandakummar C and Ganatra S (1989) Human fertilization by microinjection of immotile sperm Human Reproduction 4 175-179

Courtot AM, Jeulin C, Escalier D, Serres C, Feneux D and Jouannet P (1985) Ability of human spermatozoa without dynein arms to penetrate zona-free hamster oocytes Human in vitro Fertilization INSERM Symposium 24, pp 145-148 Eds J Testard and R Frydman. Elsevier Science Publishers, Amsterdam

David G, Bisson JP, Czyglik F, Jouannet P and Gernigon P (1975) Anomalies morphologiques du spermatozoïde humain. I. Proposition pour un system de classification Journal de Gynecologie Obstétrique et de Biologie de la Reproduction 4 (Supplement 1) 17-43

David G, Feneux D, Serres C, Escalier D and Jouannet P (1993) Une nouvelle entité pathologique du spermatozoïde: la dyskinésie flagellaire périaxonémale Builletin de l'Academie Nationale de Médecine 177 263-275

David G, Serres C and Jouannet P (1981) Kinematics of human spermatozoa Gamete Research 4 83-95

Escalier D and David G (1984) Pathology of the cytoskeleton of the human sperm flagellum: axonemal and periaxonemal anomalies Biology of the Cell $\mathbf{5 0}$ $37-52$
Escudier E, Escalier D, Pinchon MC, Boucherat M, Bernaudin JF and Fleury-Feith H (1990) Dissimilar expression of axonemal anomalies in respiratory cilia and sperm flagella in infertile men American Review of Respiratory Disease $\mathbf{1 4 2}$ $674-679$

Feneux D, Serres C and Jouannet P (1985) Sliding spermatozoa: a dyskinesia responsible for human infertility Fertility and Sterility 44 508-511

Jouannet P, Escalier D, Serres C and David G (1983) Motility of human sperm without outer dynein arms Journal of Submicroscopic Cytology 15 67-71

Katz DF, Drobnis EZ and Overstreet JW (1989) Factors regulating mammalian sperm migration through the female reproductive tract and oocyte vestments Gamete Research 22 443-469

Klemm M and Engel W (1991) Subzonal microinjection of mouse spermatozoa: insufficient sperm motility might induce phagocytosis Molecular Reproduction and Development 28 47-54

Kobayashi K, Okuyama M, Fujimoto G, Rothman C, Hill D and Ogawa S (1992) Subzonal insemination with a single spermatozoon using manipulationassisted sperm adhesion onto the ooplasmic membrane in mouse ova Molecular Reproduction and Development $31223-229$

Mortimer D, Pandya IJ and Sawers RS (1986) Relationship between human sperm motility characteristics and sperm penetration into human cervical mucus in vitro Journal of Reproduction and Fertility 78 93-102

Serres C, Feneux D and Jouannet P (1986) Abnormal distribution of the periaxonemal structures in a human sperm flagellar dyskinesia Cell Motility and Cytoskeleton 6 68-76

Wolf JPh, Feneux D, Escalier D, Rodrigues D, Frydman R and Jouannet P (1993) Pregnancy after subzonal insemination with spermatozoa lacking outer dynein arms Journal of Reproduction and Fertility 97 487-492

Zouari R, De Almeida M, Rodrigues D and Jouannet P (1993) Localization of antibodies on spermatozoa and sperm movement characteristics are good predictors of in vitro fertilization success in cases of male autoimmune infertility Fertility and Sterility 59 606-612 